index •comunicación | no 11(2) 2021 | Páginas 13-20

E-ISSN: 2174-1859 | ISSN: 2444-3239 | Depósito Legal: M-19965-2015

Recibido el 01_07_2021 | Aceptado el 07_07_2021 | Publicado el 15_07_2021

\title{
GAME STUDIES, NEXT LEVEL? \\ NUEVOS HORIZONTES PARA \\ UNA DISCIPLINA EMERGENTE
}

GAME STUDIES, NEXT LEVEL?

NEW HORIZONS FOR AN EMERGING DISCIPLINE

https://doi.org/10.33732/ixc/11/02Gamest

Salvador Gómez-García

Universidad de Valladolid

salvadorgomez@hmca.uva.es

https://orcid.org/0000-0001-5126-6464

Teresa de la Hera

Erasmus University of Rotterdam

delahera@eshcc.eur.nl

https://orcid.org/0000-0002-5102-813X

Alfonso Cuadrado-Alvarado

Universidad Rey Juan Carlos

alfonso.cuadrado@urjc.es

https://orcid.org/0000-0001-9192-5030 
14

Resumen: Tras fraguarse durante las últimas décadas, la disciplina de Game Studies ha concluido su fundamentación teórica y metodológica y ha desarrollado un interés institucional por parte de académicos de todo el mundo. Este número temático de la revista index.comunicación presenta una recopilación de estudios relacionados con la disciplina de Game Studies, llevados a cabo en el ámbito español y centrados en la exploración de líneas de futuro de una disciplina heterogénea y transversal. En concreto, se pone el foco en rasgos de las particularidades narrativas del juego, su potencial persuasivo y su influencia social. El principal objetivo de este especial es consolidar un punto de reflexión de la investigación de presente y de futuro sobre los Game Studies.

Palabras clave: Game Studies; juegos digitales; ludonarrativa; juegos serios; videojuegos.

Abstract: After being forged over the last decades, the discipline of Game Studies has completed its theoretical and methodological foundations and has developed an institutional interest on the part of academics from all over the world. This thematic issue of the journal index.comunicación presents a compilation of studies related to the discipline of Game Studies, carried out in Spain and focused on the exploration of the future of a hetero-heterogeneous and transversal discipline. Specifically, the focus is on features of the narrative characteristics of games, their persuasive potential and their social influence. The main objective of this special issue is to consolidate a point of reflection on the present and future research on Game Studies.

Keywords: Game Studies; Digital Games; Ludonarrative;

Serious Games; Videogames. 


\section{Introducción}

La publicación de este monográfico coincide con el veinte aniversario de Game Studies, la publicación decana de esta disciplina. Su rol fundacional en 2001 se fundamentó en su interés por explorar «aspectos estéticos, culturales y comunicativos de los juegos de ordenador, pero [también] cualquier artículo inédito centrado en los juegos y la ludificación» («About Game Studies»: para. 3). De forma reciente, su editor jefe, Espen Aarseth, celebraba el veinte aniversario de la revista concluyendo que la situación actual de los Game Studies «nos demuestra que habíamos estado en el buen camino, y que ya no estábamos solos» (Aarseth, 2021: para. 9).

Este editorial refleja un recorrido y el pulso de una disciplina joven académicamente, pero consolidada en su conceptualización, teorías y métodos (Siitonen, De la Hera \& Reer, 2021). Una situación reconocida por algunas de las principales asociaciones académicas internacionales como la European Communication Research and Education Association (ECREA), la International Communication Association (ICA) y la National Communication Association (NCA), que han impulsado subsecciones propias de Game Studies o Digital Games Research.

En elámbito español, este proceso sigue en vías de construcción, aunque con avances significativos en los últimos años. El reciente anuncio del capítulo hispano de DIGRA, la asociación más relevante de investigación en juegos digitales, señala nuevos pasos en la consolidación de los Games Studies regionales. Su congreso fundacional «El mapa y el juego. Los Game Studies en España ${ }^{1}$ » permitirá potenciar las miradas locales de esta disciplina en nuestro país.

Esta popularidad de los Game Studies en el contexto español no plantea un fenómeno novedoso por sí mismo, pero sí increíblemente activo en los últimos años. A un conjunto de tesis doctorales y obras fundacionales en castellano que reconocían la particularidad del fenómeno fuera de ámbitos más habituales como la educación (Cuadrado, 2007; Pérez, 2012; Navarro Remesal, 2013; Planells, 2013), se han sumado monográficos temáticos en revistas (Gonzalo-Iglesia et al., 2018; Martín \& Navarro, 2020), nuevas propuestas en editoriales consolidadas (Cuadrado \& Planells, 2020; Venegas, 2020; Moreno \& Gómez, 2021), y la aparición de colecciones temáticas en editoriales solventes como la colección Game Studies de la editorial Héroes de Papel (p.e. Velasco \& Flores, 2020) o la colección Ludografías de Shangrila (p.e. Venegas \& Moreno, 2021). Las referencias anteriores no agotan, obviamente, las aportaciones a un

\footnotetext{
1 Este evento se celebrará en diciembre de 2021 y la información del congreso se puede localizar en https://videojocs.tecnocampus.cat/digraes21/
} 
fenómeno de mayor alcance, pero sí permiten señalar su excepcional vitalidad en los últimos años en nuestro país, que se acerca a los estándares internacionales de esta disciplina. Un conjunto de líneas y visiones que han confluido en la obra colectiva Pensar el videojuego. 25 caminos para los Game Studies (Navarro, 2020) que aúna las perspectivas de un conjunto de investigadores españoles.

La confluencia de estos elementos fundamenta este monográfico. Nuestra propuesta original era ofrecer una oportunidad para explorar el futuro de una disciplina heterogénea y transversal. Los artículos que se presentan a continuación no son una excepción a esta regla y destacan por reflejar algunas de las líneas de fuerza actuales de los Game Studies.

\section{Artículos de este monográfico}

En el primer artículo de este número temático, Rodríguez-Ramírez, Arbaiza y Yalán (2021) han analizado cómo el in-game advertising (IGA) se incrusta en los juegos digitales. Se parte de conceptos clásicos como el product placement (Baños \& Rodríguez, 2012), advergames (De la Hera, 2019) o in-game advertising (Ríos-Pino et al., 2021) para concretar el rol de la marca ADIDAS en FIFA20 de EA Sports y su percepción por parte de un grupo de jugadores peruanos. La investigación emplea una metodología de encuestas y profundiza tanto en la experiencia del usuario con el videojuego (FIFA20) como en la experiencia visual de la marca (ADIDAS). Los resultados evidencian una relación complementaria que nutre la construcción de la imagen de marca a través de refuerzos positivos dentro del juego. La investigación, como señalan sus autores, se ha visto lastrada por la emergencia sanitaria producida por la Covid-19 y convendrá que, algunos de sus resultados, vuelvan a ser evaluados de forma presencial.

La investigación anterior subraya la importancia de los elementos narrativos para cumplir los propósitos de los juegos digitales. Una línea que explora Alberto Porta (2021) en su análisis sobre The Awesome Adventure of Captain Spirit (Dontnod Entertainment, 2018) combinando diferentes aproximaciones ludonarrativas que investigan la definición de la libertad del juego digital a partir de las acciones y mecánicas disponibles para el jugador (Martín-Núñez \& Planes-Cortell, 2015). De esta forma, el artículo profundiza en rasgos del lenguaje videolúdico, defendiendo la estrecha vinculación entre los rasgos narrativos y lúdicos de los juegos digitales. En el caso de estudio que se propone se potencia el análisis de dos tipos de relaciones: el jugador con el juego (o con el producto que ha creado un diseñador) y el jugador con su avatar. En ambos casos, la idea que envuelve esta investigación reside en cómo este 
videojuego trata de introducirnos en el mundo imaginario de un niño y en la construcción de la empatía, por parte del jugador, en ese proceso.

Una suma lógica de las dos primeras investigaciones se localiza en la propuesta de Martín y Pedrero (2021). La popularidad de los deportes electrónicos y su expansión social y económica ha oscurecido, en ocasiones, la naturaleza ludonarrativa de sus propuestas. Una carencia que esta investigación trata de enmendar desde la perspectiva del consumo como espectáculo de una de las propuestas más populares: League of Legends. De este modo, los autores inician una prolija aproximación histórica a la fundamentación del fenómeno de los deportes electrónicos para subrayar una capa narrativa oscurecida por la dimensión competitiva y espectacularizada que, en el caso de League of Legends, se ha visto revitalizada por Riot, empresa desarrolladora de este juego digital. El objetivo final de esta estrategia narrativa es obtener una mayor fidelización por parte de la audiencia que consume este tipo de contenidos. Los autores consiguen centrar esta dimensión del videojuego como espectáculo de masas y concluyen con una serie de reflexiones sobre los aspectos más explotados e infrautilizados por parte de Riot a la hora de promocionar el juego.

El potencial narrativo para la construcción de experiencias significativas es el punto de partida de Manzano-Zambruno y Paredes-Otero (2021) a la hora de identificar la propuesta de sensibilización contra la violencia de género que propone el análisis de los juegos The Kite, Little Kite y Repentant desarrollados por la productora ucraniana Anate Studio. Para ello, se sigue un enfoque ludonarrativo que maneja la construcción de estereotipos y su complejidad para evaluar la eficacia de la propuesta. El análisis presenta algunos de los retos más acuciantes de los juegos digitales cuando se enfrentan a cuestiones espinosas y muy sensibles socialmente. Por un lado, la ausencia de una conceptualización de la violencia de género que está muy presente en esta trilogía de juegos y en la que - según la propuesta de los autores- debería haber un mayor compromiso con su concienciación y erradicación. Un arduo debate sobre el compromiso o la actuación que deben tener aquellos juegos que abordan temas de gran complejidad social.

La aportación que cierra este monográfico manifiesta la ambición de ofrecer un primer conjunto de claves para el análisis y comprensión de los mundos abiertos como construcciones exclusivas de los juegos digitales. La propuesta de García Moreno (2021) reconoce la vastedad de estos entornos digitales e intenta ofrecer claves ludonarrativas para el análisis de estas construcciones. Para ello, se tienen en cuenta estudios de caso particulares para ofrecer un modelo teórico fundamentado en el diseño de la experiencia de 
juego, la experiencia narrativa y la construcción de mundos simulados que funcionan como sistemas. Un ambicioso modelo de análisis en el que GarcíaMoreno combina diferentes elementos y ofrece una aproximación a las implicaciones que cada elemento estético, lúdico o narrativo suponen para la experiencia del jugador en los vastos entornos de los mundos abiertos. Posteriormente se prueba la eficacia a través del mundo abierto desarrollado por Hideo Kojima en Death Stranding (2019).

\section{3. ¿Próximo nivel o próxima generación? Mirando hacia el futuro de los Game Studies}

Los artículos que forman parte de este monográfico ofrecen un retrato de algunas de las líneas de evolución de los Game Studies en la actualidad. La principal es la reflexión sobre la capacidad del juego de superar sus límites naturales —el, en ocasiones denostado, «círculo mágico» de Huizinga (1938)— para incorporar su discurso a nuestra percepción de elementos de la vida cotidiana. También se puede subrayar el interés por la narrativa específica de este medio tanto en el análisis de experiencias de éxito como en nuevas propuestas metodológicas para manifestar su especificidad. Por último, y rodeando gran parte de estas aportaciones, la transversalidad que envuelve a los Game Studies que, en ocasiones, son un fin en sí mismo o medio para investigaciones desde el marco de otras disciplinas.

El espacio de los Game Studies como disciplina de conocimiento no debe de verse monopolizado por la fuerza de las industrias culturales y sus dinámicas de negocio. La investigación necesita de una distancia y reflexión que no tiene el devenir del mercado. Para desarrollarse y ensanchar su área los Game Studies deben de mirar en varios ejes. El primero hacia el legado cultural de lo que significa el juego. Una actividad universal que no ha tenido la suficiente atención como parte de la cultura con mayúsculas y han pasado desapercibidas sus complejas y ricas implicaciones en la ficción, el arte y las actividades sociales, siendo arrinconado a espacios muy acotados dentro de la esfera educativa de la infancia. Los Game Studies tienen que reivindicar este legado, ahondar en su conocimiento y proyectarlo a otro eje, que se expande de forma circular hacia otras disciplinas, pues los juegos analógicos y digitales atraviesan nuestra contemporaneidad en aspectos filosóficos, puramente lúdicos, sociales, educativos, etc. Y, por ultimo, el tercer eje es el que se proyecta al futuro. En un espacio social donde la tecnología impregna las relaciones sociales, el trabajo y el consumo, los Game Studies deben de reivindicar una nueva visión donde lo digital no pierda su humanidad, para convertirse en 
humanidades digitales, una esfera de integración del conocimiento pues solo la hibridación es la clave de la constante renovación cultural.

Nos queda concluir con la esperanza de haber propiciado un espacio de debate y reflexión que haya permitido desentrañar y difundir mejor los Power-Ups ocultos del actual nivel de la investigación en Game Studies y, ojalá, algunos de los rasgos de una futura generación de investigaciones.

\section{Referencias bibliográficas}

AARSETH, E. (2021). Two decades of Game Studies. Game Studies, 21(1). http://gamestudies.org/2101/articles/aarseth_anniversary

BAÑOS GONZÁLEZ, M. y RODRÍGUEZ GARCÍA, T.C. (2012). Imagen de marca y product placement. ESIC.

CUADRADO, A. (2007). Los personajes virtuales: diseño, personalidad e interacción en el videojuego de los Sims 2. [Tesis doctoral]. Universidad Complutense de Madrid.

CuAdRADO, A. y Planells, A.J. (2020). Ficción y videojuegos. Teoría y práctica de la ludo-narración. UOC.

DE LA HERA, T. (2019). Digital gaming and the advertising landscape. Amsterdam University Press.

https: / / doi.org/10.5117/9789462987159

GARCíA MoRENO, R. (2021). Videojuegos de mundo abierto: propuesta de un modelo de análisis ludonarrativo. index.comunicación, 11(2), 109-132. https: //doi.org/10.33732/ixc/11/Videoj

GonZALO-IGLESIA, J.L.; Planells DE LA MAZA, A.J. y NAVARRo-REMESAL, V. (2018). Game studies today: In and beyond digital culture. Catalan Journal of Communication \& Cultural Studies, 10(1), 155-159.

https: //doi.org/10.1386/cjcs.10.2.155_2

HuizingA, J. (1938) Homo Ludens. Amsterdam University Press.

LEDESMA, A.F. y PADIAL, P.V. (Eds.) (2020). Ideological Games: Videojuegos e ideología. Héroes de Papel Studies.

MANZANO-ZAmbruno, L. y PAREDES-OTERo, G. (2021). 'Serious games' y violencia de género. Un análisis lúdico-narrativo de la trilogía 'The Kite'. index.comunicación, 11(2), 81-107. https: //doi.org/10.33732/ixc/11/Seriou

MARTín, D. y PEDRERO, L.M. (2021). Deporte y espectáculo en la narrativa de los 'e-sports': el caso de 'League of Legends'. index.comunicación, 11(2), 59-79. https: //doi.org/10.33732/ixc/11/Deport

MARTín NÚÑEZ, M. y NAVARRO REMESAL, V. (2021). La complejidad ludonarrativa en el videojuego: un doble boomerang. L'Atalante. Revista De Estudios CinematográFicos, O(31). 
MARTín-NúÑEZ, M. y Planes-Cortell, C. (2015). Las mecánicas significantes para el diseño narrativo de los videojuegos. En MARTínEZ DE SALAZARMuÑoz, I. y Alonso-URBANO, D. (eds.). Videojuegos: diseño y sociología. Editorial ESNE.

Moreno Cantano, A.C. y Gómez García, S. (Eds.) (2021). Videojuegos del presente. La realidad en formato lúdico. Trea.

NAVARRO-REMESAL, V. (2015). ¿La vida empieza o acaba con el matrimonio? Amor, diseño de ética y libertad dirigida en Catherine. adComunica. Revista Científica de Estrategias, Tendencias e Innovación en Comunicación, 9, 4361. http: / / dx.doi.org/10.6035/2174-0992.2015.9.4

NAVARRO-REMESAL, V.M. (2016). Libertad dirigida. Una gramática del análisis y diseño de videojuegos. Shangrila Ediciones.

NaVARro-RemeSAL, V.M. (Ed.) (2020). Pensar el juego. 25 caminos para los Game Studies. Shangrila Ediciones.

PÉrEZ-LATORre, Ó. (2012). El lenguaje videolúdico. Análisis de la significación del videojuego. Laertes.

Planells, A. J. (2015). Videojuegos y mundos de ficción: De “Super Mario” a "Portal". Cátedra.

PorTA, A. (2021). Con Captain Spirit somos tres. sobre mecánicas y elecciones significantes. index.comunicación, 11(2), 41-58. https: / / doi.org/10.33732/ixc/11/02Concap

Ríos-Pino, L.F.; MejíA-PEREA, J.E. y GALLARDo-EchEnique, E.E. (2021). Players Attitudes Towards In-Game Advertising. In: A. RoCHA., C. FERRÁS, P.C. LÓPEZLóPEZ, T. GUARDA (eds.), Information Technology and Systems. ICITS 2021. Advances in Intelligent Systems and Computing, v. 1330. Springer, Cham. https: / / doi.org/10.1007/978-3-030-68285-9_17

RodríGuEZ-RAMÍREZ, R.; ARBAIZA, F. y YALÁN, E. (2021). Percepción de 'gamers' universitarios de la UPC sobre el 'in-game advertising' de Adidas en el videojuego FIFA20. index.comunicación, 11(2), 21-40.

https: / /doi.org/10.33732/ixc/11/02Percep

SiITONEN, M.; DE LA HERA, T. \& REER, F. (2021). Looking ahead in games research: Entry points into a pragmatic field of inquiry. Media and Communication, 9(1), 1-4. https://doi.org/10.17645/mac.v9i1.3685

Venegas, A. (2020). Pasado interactivo. Memoria e historia en el videojuego. Sans Soleil Ediciones.

Venegas Ramos, A. y Moreno Cantano, A. C. (2021). Protestas interactivas. El videojuego como medio de reivindicación política y social. Shangrila Ediciones. 